\title{
Staphylococcus aureus nasal carriage and its antibiotic resistance profiles in children in high altitude areas of Southwestern China
}

\author{
Zongrong Gong, M.D. ${ }^{a}$, Min Shu, PH.D. ${ }^{a}$, Qing Xia, M.D. ${ }^{b}$, Shan Tan, M.D. ${ }^{a}$, Wei Zhou, M.D. ${ }^{c}$, \\ Yu Zhu, PH.D. ${ }^{a}$ and Chaomin Wan, PH.D. ${ }^{a}$
}

\begin{abstract}
Background/Aim. To describe the epidemiological profile of nasal carriage of Staphylococcus aureus (S. aureus) strains, its antibiotic resistance and $m e c A$ and Panton Valentine leukocidin (PVL) genes presence, in schoolchildren residing in highaltitude areas of Southwestern China.

Methods. The cross sectional study screened nasal swabs taken from students for S.aureus. PCR was performed to identify mec $A$ and PVL genes.

Results. Of the total 314 children $5.10 \%(16 / 314)$ was detected S.aureus. The resistance of isolated strains to penicillin, erythromycin, clindamycin, rifampicin and cefoxitin was $100 \%, 81.3 \%, 81.3 \%, 0.0 \%$, and $6.3 \%$ respectively. No strains demonstrated resistance to vancomycin; expression of mecA gene was detected in 3 isolates and 10 isolates were PVL-positive. Conclusion. S. aureus was detected in $5.10 \%(16 / 314)$ of the study population; $0.96 \%(3 / 314)$ had methicillin resistant S.aureus (MRSA); expression of the mecA and PVL genes were detected in 3 and 10 isolates respectively.
\end{abstract}

Key words: antibiotic resistance; child; Staphylococcus aureus.

http:/ / dx.doi.org/10.5546/ aap.2017.eng.274

To cite: Gong Z, Shu M, Xia Q, et al. Staphylococcus aureus nasal carriage and its antibiotic resistance profiles in children in high altitude areas of Southwestern China. Arch Argent Pediatr 2017;115(3):274-277.

a. Department of Pediatrics, West China Second Hospital, Sichuan University, Chengdu 610041,PR China, Key Laboratory of Birth Defects and Related Diseases of Women and Children, Ministry of Education (Sichuan University).

b. Department of Pediatrics, the People' s Hospital of Aba, Maerkang 624000, PR China.

c. Department of Clinical Laboratory, West China Second Hospital, Sichuan University, Chengdu 610041, PR China.

E-mail address: Chaomin Wan, PH.D.: wcm0220@126.com

Funding: Financial grant was provided by the National Natural Science Foundation of China (Grant No.81102195/H2610) and a project by Department of Science and Technology of Sichuan Province (Grant No.2012JY008).

Conflict of interest: None.

Received: 7-15-2016

Accepted: 12-5-2016

\section{INTRODUCTION}

Staphylococcus aureus (S. aureus) is an important pathogen that affects children worldwide, and the prevalence of methicillin-resistant Staphylococcus aureus (MRSA) is increasing. ${ }^{1}$ One report found that about $80 \%$ of S. aureus infections were caused by the nasal carriage of $S$. aureus. ${ }^{2}$ Resistance to $\beta$-lactam antibiotics is conferred by penicillinbingding protein encoded by the mec $A$ gene which is located on a large chromosomal element.

Recently, MRSA has been increasingly identified as the major cause of communityonset infections, usually possing Panton Valentine leukocidin (PVL) genes. For condition in high altitude, in climate from the extreme environments such as high UV and cold are believed that bacteria develop efficient mechanisms and strategies to growth and survive. ${ }^{3}$ What's more, the spread of bacteria resistant depends on the community of people and antibiotic usage to some extent.

In China, There are about 250 million square kilometers in high altitude and the population about 130 million, so it's essential to know about the prevalence of $S$. aureus and it's antibiotic resistance in the area. The population density and access to healthcare in Tibetan regions is lower than mainland China. In some ways, it's bad for growth and spread of $S$. aureus. In China, most studies ${ }^{4,5}$ were performed among children in mainland and only one of our studies ${ }^{6}$ was about healthy school children 8 years ago. However, with the traffic and healthcare development, the epidemiology of $S$. aureus may be changed. The purposes of this study was to describe the prevalence of $S$. aureus, its antibiotic resistance and mecA and PVL genes presence, in school children residing in high altitude areas of Southwestern China.

\section{POPULATION AND METHODS}

This study was conducted between October and November of 2012 in Maerkang City (altitude ranging between 2500 and 4100 meters); a city of Aba province with a population density of 
about 9 people per square kilometer. In this rural region, access to medical resources is relatively scarce. Antibiotics such as penicillin, clindamycin, erythromycin are sometimes used, though most other antibiotics are not commonly used in this population. In this study, we calculated the minimum sample size required to accurately assess the incidence of S.aureus carriage in the region as 233 , based on the expected prevalence of nasal colonization $(30 \%),{ }^{7}$ with a $95 \%$ confidence interval.

Healthy Tibetan children were recruited from primary schools in Maerkang City. Children receiving antibiotic therapy or requiring hospital admission within a 4 week period preceeding the time of recruitment as well as children suffering from skin infections were excluded. Samples of swabs were collected by twice rotating a sterile cotton swab pre-wetted with sterile saline solution in the vestibule of both anterior nares of the participants after obtaining oral, parental informed consent.

Nasal swabs were transported to the laboratory using the Sterile Amies Agar Gel Transport Swab (Copan, Italy) and inoculated on the mannitol salt agar (Oxoid Ltd, Basingstoke, United Kingdom) and Columbia blood agar (bioMérieux, France) for $6 \mathrm{~h}$. Next, the inoculations were incubated in $5 \% \mathrm{CO}_{2}$ for $16-24 \mathrm{~h}$ at $35^{\circ} \mathrm{C}$. The identification of S.aureus was based on colony morphology, positivity of catalase and tube coagulase, and the results of a Slidex Staph Plus kit (bioMérieux, France). Antibiotic susceptibility of the isolates was performed by disc diffusion method, in accordance with the Clinical and Laboratory Standards Institute (CLSI) recommendations, for the following antibiotics: penicillin, erythromycin, clindamycin, gentamicin, cefoxitin and vancomycin. All the isolated S.aureus strains were screened for the mecA gene by PCR to identify a $162 \mathrm{bp}$ target product. Primers used were mec $A$ P4 5'-TCCAGATTACAACTTCACCAGG-3' and mecA P 7 5'-CCACTTCATATCTTGTAACG-3' ${ }^{8}$ All isolates were also assayed for the presence of PVL genes by PCR using the luk-pv1:5'-ATCATTAGGTAAAA TGTCTGGACATGATCCA-3' and luk-pv2: 5'-GCATCAACTGTATTGGATAGCAAAAGC-3' primers. ${ }^{9}$ Only strains harbouring the mec $A$ gene were classified as MRSA. ${ }^{10}$

The chi-square tests or Fisher' s exact test were performed to detect the statistical significance (5\%) between groups using SPSS 13.0 software for windows (SPSS Inc, Chicago, IL, United
States of America). Ethical approval for this study was approved by the medical committee of West China Secondary Hospital, Chengdu, on 28 February 2008.

\section{RESULTS}

Three hundred and fourteen healthy Tibetan children including $182(58.0 \%)$ boys and 132 $(42.0 \%)$ girls participated in the study. The average age was $10.2 \pm 1.2$ years (ranging from 6 to 11 years) with $44.6 \%$ being above 10 years. S. aureus strains were isolated from $16(5.10 \%)$ children including 10 boys and 6 girls without significant difference with regard to gender $(p=0.706)$. The resistance of isolated strains to penicillin, erythromycin, clindamycin, rifampicin and cefoxitin was $100 \%, 81.3 \%, 81.3 \%, 0.0 \%$, and $6.3 \%$ respectively. The strain resistant to cefoxitin was proved to be MRSA. Additionally, expression of the mec $A$ gene was detected in 3 isolates and 10 isolates were PVL-positive.

\section{DISCUSSION}

S. aureus is a major human pathogen that is known to cause a broad range of serious community acquired and nosocomial diseases in humans, from minor skin infections to severe infections such as septicemia. ${ }^{4}$ The carriage of $S$. aureus can be of great importance to the development of new therapeutic strategies and more effective prevention. Here we present the prevalence and antibiotic data of $S$. aureus isolates extracted from the nasal carriage of children in high altitude areas of southwest China. To our knowledge, this is the first study reporting the prevalence of MRSA in this regional pediatric population.

A few studies have previously investigated the carriage rate of S.aureus in low-altitude regions of mainland China ${ }^{5}$. Our team assessed the prevalence of S.aureus carriage within healthy school children in Chengdu, China; it was $18.35 \%$ and MRSA accounted for about $6 \%$ of the isolated S. aureus strains among 801 healthy children. ${ }^{5}$ Our study revealed that the nasal carriage rate of $S$. aureus among Tibetan healthy children was $5.10 \%$. This rate was fairly low compared with reports from low-altitude studies. Interestingly, this rate is similar to a reported rate of lower respiratory infection $6.16 \%(22 / 357)$ in Tibetan children ${ }^{11}$ and the reported rate of $6.18 \%(11 / 178)$ cases of pediatric intensive care hospitalization ${ }^{12}$ in a high altitude area of China. The low rates of pediatric disease and S.aureus carriage in 
this region may be related to the environmental elevation in which the ambient air is thin and cold and where the population density is low. While children from areas of better economic status and greater populations (such as Chengdu) typically have greater access to medical care and receive antibiotic therapy frequently, the low rates found in our sample population could be related to lower economic states and poor medical conditions. Nonetheless, this relationship needs further determination.

In our study, the nasal carriage rate of $S$. aureus and MRSA was $5.10 \%(16 / 314)$ and $0.96 \%(3 / 314)$ in healthy children, respectively. However, another study ${ }^{6}$ by our team carried 8 years ago demonstrated that the nasal carriage rate of $S$. aureus was $2.4 \%$ in a population of 673 healthy Tibetan children, with no MRSA detected. The results show the rate of $S$. aureus is increasing in the area and attention to antibiotic usage for $S$. aureus should be considered, especially where MRSA in concerned. The results of this study indicated that in the presence of children with severe $S$. aureus infection, we should enhance antibiotics treatment based on antibiotic susceptibility test. Additional, vancomycin is needed when MRSA is detected in isolates. What's more, the prevalence of $S$. aureus is still low but increasing in healthy children. Data about S. aureus prevalence in high altitudes is scarce as typically mainland China is the focus of such investigations. One, study ${ }^{13}$ reported S.aureus led to a mortality rate of $37.0 \%$ in neonatal septicaemia in a high altitude African city. And it is important to continue investigating the environmental factors which may contribute to the incidence of pediatric $S$. aureus in these areas.

\section{Limitations of the study}

The present study had certain limitations. Due to the low population density of the region, it was difficult to recruit qualifying participants. The lack of differences between genders could be the result of a short number of patients. Additionally, the epidemiological study of the nasal carriage of $S$. aureus in this region needs to be further examined and dynamically monitored as it can be of great importance for this region.

\section{CONCLUSION}

S. aureus was detected in $5.10 \%(16 / 314)$ of the study population; $0.96 \%$ (3/314) had MRSA; expression of the mecA and PVL genes were detected in 3 and 10 isolates respectively.

\section{REFERENCES}

1. Song X, Cogen J, Singh N. Incidence of methicillin-resistant Staphylococcus aureus infection in a children's hospital in the Washington metropolitan area of the United States, 2003 - 2010. Emerg Microbes Infect 2013;2(10):e69.

2. Wertheim HF, Vos MC, Ott A, van Belkum A, et al. Risk and outcome of nosocomial Staphylococcus aureus bacteraemia in nasal carriers versus non-carriers. Lancet 2004;364(9435):703-5.

3. Dib J, Motok J, Zenoff VF, Ordoñez O, et al. Occurrence of resistance to antibiotics, UV-B, and arsenic in bacteria isolated from extremeenvironments in high-altitude (above 4400 m) Andean wetlands. Curr Microbiol 2008;56(5):510-7.

4. David MZ, Daum RS. Community-associated methicillinresistantStaphylococcus aureus:epidemiology and clinical consequences of an emerging epidemic. Clin Microbiol Rev 2010;23(3): 616-87.

5. Fan J, Shu M, Zhang G, Zhou W, et al. Biogeography and virulence of Staphylococcus aureus. PLoS One 2009;4(7):e6216.

6. Deng JJ, Xiao GG, Zhu Y, Zhou W, etal. Staphylococcus aureus Nasal Carriage and Its Antibiotic ResistanceProfiles in TibetanSchoolChildren in Southwest China. HKJPaediatr (new series) 2014;19:75-8.

7. Lamaro-Cardoso J, de Lencastre H, Kipnis A, Pimenta FC, et al. Molecular epidemiology and risk factors for nasal carriage of staphylococcus aureus and methicillin-resistant S. aureus in infants attending day care centers in Brazil. J Clin Microbiol 2009;47(12):3991-7.

8. Oliveira DC, de Lencastre H. Multiplex PCR strategy for rapid identification of structural types and variants of the mecelementin methicillin-resistantStaphylococcus aureus. Antimicrob Agents Chemother 2002;46(7):2155-61.

9. Lina G, Piémont Y, Godail-Gamont F, Bes M, et al. Involvement of Panton-Valentine leukocidin-producing Staphylococcus aureus in primary skin infections and pneumonia. Clin Infect Dis 1999;29(5):1128-32.

10. Stegger M, Andersen PS, Keams A, Pichon B, et al. Rapid detection, differentiation and typing of methicillin-resistant Staphylococcus aureus harbouring either mecA or the new mecA homologue mecA(LGA251). Clin Microbiol Infect 2012;18(4):395-400.

11. XueH. Pathogenic analysis of Tibetan children in acutelow respiratory tract infection in high-altitude areas. Zhongguo Xian Dai Yi Sheng 2015(26):76-8.

12. Xia YL, Ge M, Wang Z. [Pathogenic analysis of ventilatorassociated pneumonia in the pediatric intensive care unit in high-altitude areas]. Zhongguo Dang Dai Er Ke Za Zhi 2014;16(8):787-90.

13. Airede AI. Neonatal septicaemia in an African city of high altitude. J Trop Pediatr 1992;38(4):189-91. 